\title{
DEEP2 Trial - Key Findings in Paediatric Patients with Transfusion-dependent Thalassaemia
}

An Expert Interview with Antonis Kattamis on behalf of the DEEP Consortium

Division of Pediatric Hematology-Oncology, First Department of Pediatrics, National \& Kapodistrian University of Athens, Greece

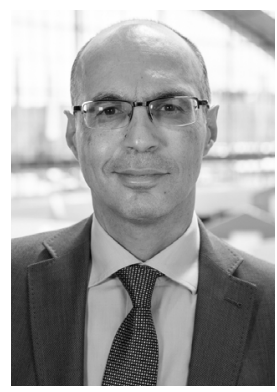

\section{Antonis Kattamis}

Antonis Kattamis is Professor of Paediatric Haematology-Oncology at the National \& Kapodistrian University of Athens, Greece. He has a specialist interest in haemoglobinopathies and has published extensively in this area, with several contributions towards improving the management of transfusion-related iron overload. Professor Kattamis is President of the Advisory Committee of Thalassemia and Haemophilia for the Central Health Committee (KESY) as part of the Greek Ministry of Health, and is President of the Hellenic Society of Paediatric Haematology and Oncology.

\section{Keywords}

Anaemia, DEEP-2, deferiprone, deferasirox, transfusion-dependent thalassaemia

Disclosures: Antonis Kattamis has received study support, speaker fees and is a member of the advisory board for Novartis Oncology, and has received speaker fees from Apopharma.

Acknowledgement: The DEEP study was funded by the EC-FP7 Framework Program. Medical writing assistance for this article was provided by Dr CG Griffin of Griffin Scientific Ltd on behalf of Touch Medical Media and was supported by Touch Medical Media.

Review Process: This is an expert interview and as such, has not undergone the journal's standard peer review process.

Compliance with Ethics: This article is an interview and did not involve any studies with human or animal subjects performed by any of the authors.

Authorship: The named author meets the International Committee of Medical Journal Editors (ICMJE) criteria for authorship of this manuscript, takes responsibility for the integrity of the work as a whole, and has given final approval for the version to be published.

Received: 17 August 2019

Accepted: 15 December 2019

Citation: European Oncology \& Haematology. 2019; 15(2):89-90

Corresponding Author: Antonis Kattamis, Division of Pediatric Hematology-Oncology, First Department of Pediatrics, National and Kapodistrian University of Athens, 'Aghia Sophia' Children's Hospital Papadiamantopoulou and Levadias, Goudi 11527, Athens, Greece. E: ankatt@med.uoa.gr

Support: No funding was received for the publication of this article.
$\mathrm{B}$ eta-thalassaemia is a hereditary blood disorder arising from impaired synthesis of beta globin chains, and can result in a range of outcomes from asymptomatic carriers to severe anaemia. ${ }^{1}$ Symptomatic thalassaemia presents in around 1 in 100,000 people worldwide, and 1 in 10,000 in Europe. ${ }^{1}$ Transfusion-dependent beta-thalassaemia (TDT) is the most severe form of thalassaemia requiring lifelong management with regular red blood-cell transfusions. Iron overload following blood transfusions is a common challenge in management and contributes to elevated risk of cardiac disease, along with complications of the liver and endocrine systems. ${ }^{1,2}$ Iron chelation is an essential component of the management of TDT in paediatric and adult population. ${ }^{2,3}$ Data supporting the role of iron chelation in younger patients with TDT are scarce; however, several studies conducted recently are providing new evidence to help inform treatment selection. ${ }^{4,5}$

\section{Q. What are the key challenges in administering lifelong red blood-cell transfusions in patients with transfusion-dependent beta-thalassaemia?}

Patients with TDT must manage chronic, potentially fatal anaemia, and require a treatment strategy of lifelong red blood-cell transfusions to provide functioning blood cells. However, each transfusion is associated with the introduction of high levels of iron, which can quickly lead to severe iron overload.

\section{Q. How have recent therapeutic advances affected outcomes in transfusion-dependent beta-thalassaemia?}

Recent advances in treatment options have contributed to greatly improved morbidity and mortality outcomes in patients with TDT, who are now expected to live a relatively normal life, with manageable disease burden.

\section{Q. What were the aims and outcomes of your recent study, DEEP-2, in patients with transfusion-dependent beta-thalassaemia?}

DEEP-2 explored the efficacy and safety of iron chelation in paediatric patients with TDT. ${ }^{5}$ This is important because there has been a relative lack of meaningful treatment data in the paediatric population with haemoglobinopathies. The DEEP-2 study compared the effects of the two available oral iron chelating agents, deferiprone and deferasirox, over 12 months of treatment. Data from this trial should benefit treatment selection and improve outcomes in the paediatric population of patient with transfusion dependent haemoglobinopathies (TDH), namely TDT, sickle cell disease and haemoglobinopathy. 


\section{Q. What were the main efficacy findings of DEEP-2, and what factors should be considered when using iron chelators in the management of transfusion-dependent beta-thalassaemia?}

Findings from the DEEP-2 trial support the use of iron chelation in the paediatric population with TDH. More precisely, both deferiprone and deferasirox can be used in setting, as there were no significant differences in efficacy or safety profiles, at least at the per protocol analysis. In order to further improve outcomes, physicians need to encourage compliance to treatment. Furthermore, rapid and accurate dosing adjustments are important in this population of patients.

\section{Q. What can be done to further improve outcomes in this patient population}

It is important to determine how early intervention with iron chelation can be considered. Emerging data, such as the findings of DEEP-2, are providing support for initiation of iron chelation at a very early age. Once treatment commences, dose adjustment will play a key role in optimising outcomes in this paediatric population.

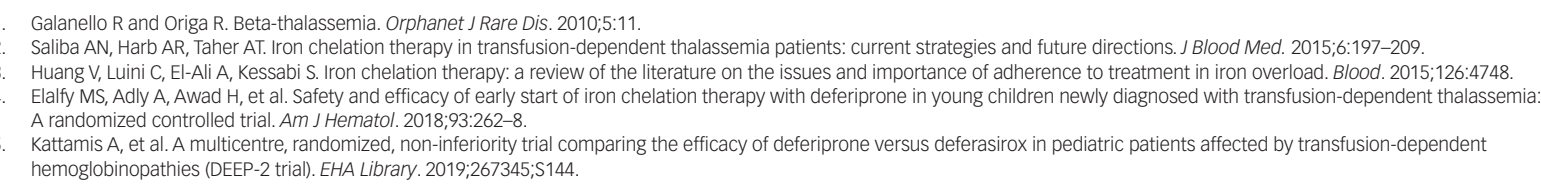

